\title{
KUNSTGESCHICHTE UND REPRÄSENTATION - ZUR TERMINOLOGIE UND FORSCHUNGSGESCHICHTE
}

\author{
WERNER TELESKO
}

Der Begriff "Repräsentation“ wurde und wird in der kunstgeschichtlichen Literatur sehr häufig und mit unterschiedlichsten Konnotationen verwendet. Dabei ist in der Regel nicht jene begriffliche Schärfe festzustellen, die den Umgang mit dem Themenkomplex Repräsentation in anderen Disziplinen (Philosophie, Rechtswissenschaft) auszeichnet. Dieser Umstand hat sicher auch mit der Tatsache zu tun, dass künstlerisches Handeln in recht allgemeiner Weise als (Wieder-)Vergegenwärtigung gesehen wird. Das Präfix re- des Wortes zeigt zudem an, dass etwas, das präsent war und es nicht mehr ist, zu einem bestimmten Zeitpunkt re-präsentiert wird. Das bekannteste Beispiel für diesen Sachverhalt ist die "equalitas"-Darstellung, die einen abwesenden Herrscher lebensgroß im Bild repräsentiert und damit einen Stellvertretercharakter begründet. Im deutschen Sprachgebrauch wird die Bedeutung des Wortes „Repräsentation" recht weit mit "Vorstellung ", 'Darstellung“, „Abbild“",Bild“ oder „Stellvertretung“ wiedergegeben. Dies lehrt auch ein historischer Rückblick: Die Weite der barocken Definition von Johannes Micraelius, ",Repraesentare, absens quodammodo facere praesens" (1662), also etwas Abwesendes durch etwas anderes gegenwärtig zu machen, erklärt darüber hinaus die Vielzahl an Anschlussmöglichkeiten für andere Disziplinen.

Für die Kunstgeschichte im Speziellen ist vor allem die mimetische Komponente von Repräsentation entscheidend, ist doch das Bild ein Zeichen, das ein bestimmtes Vorbild repräsentiert, und in diesem Sinn basiert jede mimetische Repräsentation auf der Ähnlichkeit von Bild und Vorbild. Neben diesem Aspekt ist in der bildenden Kunst der Verweischarakter wesentlich: Bestimmte Gegenstände repräsentieren bzw. implizieren eine Bedeutung, die außerhalb ihrer selbst liegt und - wenn man etwa an die Taube als bekanntes Symbol für Frieden oder den Heiligen Geist denkt - auf Konventionen basiert. Methodisch wurden in der Kunstgeschichte Themenbereiche dieser Art mit Hilfe von Emblematik oder Ikonologie analysiert.

Die heute omnipräsente Verbreitung des Begriffs "Repräsentation“ steht in offensichtlichem Widerspruch zu dessen Verwendung und Kategorisierung in frühneuzeitlichen Handbüchern: Da Repräsentation kein Begriff der Rhetorik ist, findet er sich demnach nicht in der darauf aufbauenden ästhetischen Begriffsbildung. Das Fehlen eines entsprechenden Eintrags in Zedlers berühmtem Universallexikon mag in dieser Hinsicht verwundern, sieht man einerseits doch die zeitgleiche höfische Kultur als Blütezeit der Repräsentation schlechthin und andererseits Descartes als Begründer des neuzeitlichen „Repräsentationalismus", der darauf basiert, dass es keine Idee geben könne, die uns nicht etwas zu repräsentieren scheint.

In einem allgemeinen und nicht fachspezifischen Sinn bedeutet Repräsentation jede wie immer geartete Repräsentation von Macht, basierend auf dem Zeremoniell als übergreifender Norm und Struktur. Die damit unweigerlich verbundene Sichtbarkeit jedes sozio-politischen Handelns bildete in der Vergangenheit einen wesentlichen Schwerpunkt kunstgeschichtlicher Repräsentationsforschung und wird auch in den Beiträgen des vorliegenden Bandes speziell in den Blick genommen. Die Kunstgeschichte hat sich in den letzten Jahrzehnten darüber hinaus bemüht, neben den vor allem um die Prädikate "majestas" und "splendor" kreisenden Forschungen zur Blütezeit des Absolutismus die spätestens ab der Mitte des 18. Jahrhunderts unaufhaltsam auf den Plan tretende "Krise“ der Repräsentation genauer in den Fokus zu nehmen. Letztere basiert zum einen Teil auf dem Ende der Analogisierung der Repräsentation mit dem göttlich verbürgten "ordo“ im Gefolge der Legitimationskrise der europäischen Monarchien, ist andererseits aber ebenso in Zusammenhang 
mit den politisch zunehmend an Bedeutung gewinnenden sozialen Schichten wie etwa dem Bürgertum zu sehen, welche die Repräsentation des Obrigkeitsstaates als bloßes "Theater" zu entlarven versuchten. Dazu kommen die grundlegenden Erfahrungen des ausgehenden 18. und beginnenden 19. Jahrhunderts, dass die im "klassischen" (an die Repräsentation gebundenen) Zeitalter (Michel Foucault) als zu- und ausreichend beurteilten Repräsentationssysteme sich angesichts der veränderten Wirklichkeit als nicht mehr adäquat erwiesen und somit Repräsentation ihre die Gesellschaft bestimmende Allgemeingültigkeit verlor. Mit diesen Fakten korrespondiert das Faktum, dass Repräsentation letztlich nur in der Sphäre von Öffentlichkeit existieren kann, und bereits im 18. Jahrhundert als Gegenbewegung zur Instrumentalisierung der Künste im Dienst von politischer Macht die an bestimmten Gattungen (Stilleben) besonders deutlich ablesbare Flucht der Kunst ins Private einsetzte. Dies zeigt, dass Repräsentation somit in der begrifflichen Genese als auch in der Bedeutungsbildung eng an die entsprechenden historischen und sozialen Entwicklungen gebunden ist.

In der neueren Repräsentationstheorie (Louis Marin) wird das eigengesetzliche Potential der bildenden Kunst deutlich stärker betont. In diesem Sinn werden die entsprechenden Werke als Agenten der Geschichte verstanden und nicht als bloße historische Illustrationen. Vor allem den Bildern wird demgemäß eine Realität schaffende Funktion zugeordnet. Damit wird das Werk als solches aus einer dienend verstandenen Abhängigkeit von dominierenden historischen und gesellschaftlichen Rahmenbedingungen herausgeholt und die Mimesis - in Abgrenzung zum Paradigma einer vermeintlich abbildenden Qualität von bildender Kunst - als rhetorische Strategie des Bildes gesehen. Die Königsporträts Ludwigs XIV. stehen bei Louis Marin gleichnishaft für die Macht des Bildes, Realitäten zu konstruieren. In diesem Sinne wird in Marins Analysen das Absolutismus-Paradigma nicht im Sinne eines politischen Deutungshorizontes gesehen, sondern primär als essentielle Eigenschaft dieser Macht der Bilder. Marin zufolge beruht die Macht des Königs darauf, dass er diese gar nicht ausüben muss, sondern vor allem durch entsprechende Werke glaubhaft versichert, jederzeit Macht ausüben zu können. In diesem Sinn braucht das Bild keinen Kontext, um zu zeigen, wofür es steht, es kann vielmehr selbst zeigen, was es „ist“. In dieser Betrachtungsweise fällt die sprechende Instanz mit dem besprochenen Objekt zusammen, es ist somit repräsentativ und präsent zugleich. Marin verabschiedet somit die mimetische Funktion aus dem Bereich der Repräsentation: Die Aufgabe des Bildes kann eben nicht in einer Nachahmung des perfekten Königs bestehen, sondern dient dazu, eine vollkommene Vorstellung vom König zu produzieren: „Ein König ist ein Portrait des Königs und nur das macht ihn zum König, denn ansonsten ist er ein Mann." (Louis Marin).

Demgegenüber stellt Hans Belting in seinen Untersuchungen die anthropologische Dimension der Repräsentation ins Zentrum. Ausgehend vom Faktum, dass die Repräsentation gesellschaftlicher Belange an der Schwelle zur Neuzeit primär Bildern übertragen wurde und diese Bilder in der Regel individuelle Körper darstellen, kommt er zum Schluss, dass jede Verkörperung im Bild eine vom Körper abgeleitete Ausübung von Repräsentation darstellt. Das Bild legt von diesem Spannungsverhältnis zwischen Körper und Artefakt in besonderer Weise Zeugnis ab, indem es entweder die Sichtbarkeit der Körper bekräftigt oder negiert. In diesem Sinn besteht in der Bildtheorie Beltings der ureigenste Sinn des Bildes darin, körperliche Präsenz gegen Repräsentation einzutauschen. Der hinter jeder Repräsentation stehende Körper ist somit Belting zufolge bereits als Bild aufgefasst und zum Bild hergerichtet.

Es fällt auf, dass Marin wie Belting hinsichtlich ihrer Beispiele stark auf bestimmte historische Erscheinungen abzielen - Marin auf das absolutistische Porträt, Belting auf die effigies am Übergang vom Spätmittelalter zur Frühen Neuzeit. Nicht ohne Grund sind es gerade diese beiden Themenfelder, die seit Ernst Kantorowicz maßgeblichen Arbeiten die kunsthistorische Forschung bestimmen. Repräsentation als umfassende kulturelle Praxis schließt aber letztlich die Breite unterschiedlichster - miteinander häufig verflochtener Gattungen von der barocken Medaille bis zum tableau vivant des 19. Jahrhunderts ein. Nicht alle daran beteiligten Medien können den gleichen Charakter von „Öffentlichkeit“ (Sichtbarkeit) für sich beanspruchen. Speziell die Forschung, die an bestimmte historische Beispiele (Residenzen und Hofbauten) gebunden ist, die 
mit der ganzen Breite von höfischen Repräsentationsformen ausgestattet sind, wird schwer mit der Anwendung einer bestimmten Methode das Auslangen finden, sondern in Ergänzung theoretischer Fragestellungen auch davon ausgehen müssen, Arbeitsziele und Forschungsperspektiven aus dem jeweiligen konkreten historischen Kontext zu entwickeln.

\section{BIBLIOGRAFIE}

Repräsentation. In: Joachim Ritter, Karlfried Gründer (Hrsg.): Historisches Wörterbuch der Philosophie. Band 8. Basel 1992, Sp. 790-853.

Repräsentation/repräsentativ. In: Karlheinz Barck (Hrsg.): Ästhetische Grundbegriffe. Historisches Wörterbuch in sieben Bänden. Stuttgart, Weimar 2003 (Reprint 2010). Band 5, S. 264-290.

Vera Beyer u. a. (Hrsg.): Das Bild ist der König. Repräsentation nach Louis Marin. München 2006.

Hans Belting u. a. (Hrsg.): Quel corps? Eine Frage der Repräsentation. München 2002.

Michel Foucault: Les mots et les choses. Une archéologie des sciences humaines. Paris 1966.

Ernst H. Kantorowicz: Götter in Uniform. Studien zur Entwicklung des abendländischen Königtums. Stuttgart 1998. Louis Marin: Das Porträt des Königs. Berlin 2005 (Paris 1981).

Johannes Micraelius: Lexicon Philosophicum. Stettin 1662 (Reprint Düsseldorf 1962).

Dominik Perler: Repräsentation bei Descartes (Philosophische Abhandlungen. Band 68). Frankfurt/M. 1996.

Johann Heinrich Zedler (Hrsg.): Grosses vollständiges Universallexicon aller Wissenschaften und Künste. 68 Bände. Halle, Leipzig 1732-1754. 
